EPJ Web of Conferences 66, 03078 (2014)

DOI: $10.1051 /$ epjconf/ 20146603078

(C) Owned by the authors, published by EDP Sciences, 2014

\title{
Recent Developments in the Experimental Nuclear Reaction Data Library EXFOR
}

\author{
Valentina Semkova ${ }^{a}$, Naohiko Otuka, Stanislav Simakov, Viktor Zerkin \\ IAEA, Nuclear Data Section, A-1400, Vienna, Austria
}

\begin{abstract}
The International Network of Nuclear Reaction Data Centres (NRDC) provides nuclear reaction data services to users through collection and compilation of experimental nuclear reaction data in the EXFOR database. The database includes neutron-induced, charged-particle-induced, and photonuclear data for projectile energies up to $1 \mathrm{GeV}$. Sophisticated search options and user-friendly retrieval interface for downloading data in different formats have been developed at IAEA Nuclear Data Section. Additional output options such as data plotting capabilities are provided as well. The database is constantly revised and extended and at present contains about 20,000 experimental works accumulated in its 40 years of history. The paper will present several recent IAEA NDS activities related to the development of the EXFOR database and retrieval system.
\end{abstract}

\section{Introduction}

Nuclear data are needed in different fields of science and technologies. Collections of experimental nuclear reaction data in databases were initiated in 1950s and 1960s at U.S. National Nuclear Data Center (NNDC), the Nuclear Energy Agency Data Bank (NEADB), the IAEA Nuclear Data Section, and the Russian Nuclear Data Centre (CJD). These centres joined together in 1968 in a Nuclear Reaction Data Centre Network (NRDC) and exchange of data was established in a common EXchange FORmat [1]. The EXFOR format was designed and developed in order to allow variety of numerical data and textual information to be transmitted in a machine-readable form. The information-identifier keywords, codes and the defined data structure also allow users to set specific criteria for search and further process of the retrieved information. The collection, compilation and dissemination of experimental nuclear reaction data have expanded ever since the Network was founded and at present the NRDC consist of 14 Nuclear Data Centres. The scope and the type of the compile data evolved as well with development of different nuclear applications from neutron-induced reactions to neutron, charged-particle and photonuclear data. Currently compilation is compulsory for neutron and light charged-particle $(\mathrm{A}<12)$ induced reactions at incident energies below $1 \mathrm{GeV}$. Heavyion $(\mathrm{A} \geq 13)$ and photon induced reaction data are also compiled on voluntary basis. Different types of data are compiled in EXFOR such as: reaction cross sections, differential data with respect to angle, partial differential data with respect to angle, resonance parameters, partial cross section data, polarization data, fission product yields, differential data with respect to angle and energy and many others. Access of users to the information in EXFOR is provided by means of World Wide Web

${ }^{a}$ Corresponding author: v.semkova@iaea.org

This is an Open Access article distributed under the terms of the Creative Commons Attribution License 2.0, which permits unrestricted use, distribution, and reproduction in any medium, provided the original work is properly cited. 
(http://www-nds.iaea.org/exfor/) or stand-alone CD-ROM distributed by NDS at requests. Retrieval system developed at NDS allows sophisticated search that include not only EXFOR but also a number of relevant databases such as Evaluated Nuclear Reaction Data (ENDFs), Evaluated Nuclear Structure and Decay Data (ENSDF), Bibliographic databases (CINDA, NSR) [2]. The paper describes the basic principles of EXFOR compilation and data retrieval as well some recent and future developments aimed at improved qualities and present-day needs in science and technologies.

\section{EXFOR compilation}

Collection, compilation and dissemination of experimental nuclear reaction data and the related bibliographic information in the EXFOR database is the main task of the International Network of Nuclear Reaction Data Centres (NRDC). The Network constitutes of worldwide cooperation of 14 data centres from 10 countries and 2 international organisations under the auspices of the International Atomic Energy Agency (Table 1):

Table 1. Nuclear Reaction Data Centres (NRDC)

\begin{tabular}{|c|c|}
\hline NDS & IAEA Nuclear Data Section, Austria http://www-nds.iaea.org \\
\hline NEA-DB & OECD NEA Data Bank, France http://www.nea.fr/databank \\
\hline NNDC & $\begin{array}{c}\text { National Nuclear Data Centre, Brookhaven National Laboratory, USA } \\
\text { http://www.nndc.bnl.gov/ }\end{array}$ \\
\hline ATOMKI & $\begin{array}{c}\text { Nuclear Reaction Data Group, Institute of Nuclear Research, Hungary } \\
\text { http://www.atomki.hu/atomki/CyclAppl// }\end{array}$ \\
\hline $\mathrm{CaJaD}$ & $\begin{array}{c}\text { Russian Nuclear Structure and Reaction Data Centre, Kurchatov Institute, } \\
\text { Russia http://www.kiae.ru/ }\end{array}$ \\
\hline CDFE & $\begin{array}{c}\text { Centre of Photonuclear Experimental Data, Moscow State University, Russia } \\
\text { http://cdfe.sinp.msu.ru }\end{array}$ \\
\hline CJD & $\begin{array}{c}\text { Russian Nuclear Data Centre, Institute of Physics and Power Engineering, } \\
\text { Russia http://www.ippe.ru/podr/cjd }\end{array}$ \\
\hline CNDC & $\begin{array}{l}\text { China Nuclear Data Centre, China Institute of Atomic Energy, China } \\
\text { http://www.ciae.ac.cn }\end{array}$ \\
\hline CNPD & $\begin{array}{c}\text { Centre of Nuclear Physics Data, Russian Federal Nuclear Centre, Russia } \\
\text { http://en-cnpd.vniief.ru/ }\end{array}$ \\
\hline JAEA-NDC & JAEA Nuclear Data Centre, Japan http://www.ndc.jaea.go.jp \\
\hline JCPRG & $\begin{array}{l}\text { Japan Nuclear Reaction Data Centre, Hokkaido University, Japan } \\
\text { http://www.jcprg.org/ }\end{array}$ \\
\hline KNDC & Korea Nuclear Data Center, Daejeon http://www.atom.kaeri.re.kr/ \\
\hline NDCPI & Nuclear Data Physics Centre of India, Mumbai, India \\
\hline UkrNDC & $\begin{array}{c}\text { Ukrainian Nuclear Data Centre,Institute for Nuclear Research, Ukraine } \\
\text { http://ukrndc.kinr.kiev.ua/ }\end{array}$ \\
\hline
\end{tabular}

The IAEA Nuclear Data Section and other centres regularly scan scientific journals, major conference proceedings, and reports to identify articles for EXFOR compilations and keep the database complete. Articles are assigned to different centres for compilation based on geographical distribution. Some of the centres are specialized in specific type of nuclear reaction data. The compilation files in form of EXFOR Entries are combined at IAEA NDS in a common EXFOR Master File and distributed within the Network. Each EXFOR entry contains bibliographic information, all information relevant to measurement techniques and analysis, and numerical data. The database is updated on a monthly basis with newly compiled and revised Entries. 
The database is also cross-checked with extensive bibliographic compilation found in the literature or some specialized databases. Such comparisons with proton-induced total reaction cross sections in Carlson's compilation [3], isotope production cross sections by light-charged particle induced reactions from Landolt-Börnstein compilation [4] etc. were carried out recently and missing articles were added to the database.

Specialized software related to compilation work, digitization of graphical images, and checking for compliances with EXFOR format have been developed and are available for downloading at the NRDC website (http://www-nds.iaea.org/nrdc/)

Quality assurance of EXFOR content is an important issue. Feedback from users, recommendations from the WPEC subgroup "Quality Improvements of the EXFOR Database (SG30)" [5] or mistakes found by users and compilers are thoroughly investigated and registered on the NRDC web site (http://www-nds iaea.org/nrdc/error/) for corrections. Quality of digitization was recently reviewed by a benchmark exercise organized by NDS. Results from the benchmark were discussed at a consultants' meeting and recommendations to software developers and compilers were distributed [6].

\section{EXFOR retrieval system}

Web services for data search and retrieval (Fig 1) have been developed at the IAEA Nuclear Data Section. The EXFOR format and coding of the information allow users to set criteria of search. List of possible codes with short description is provided for each of available search fields.

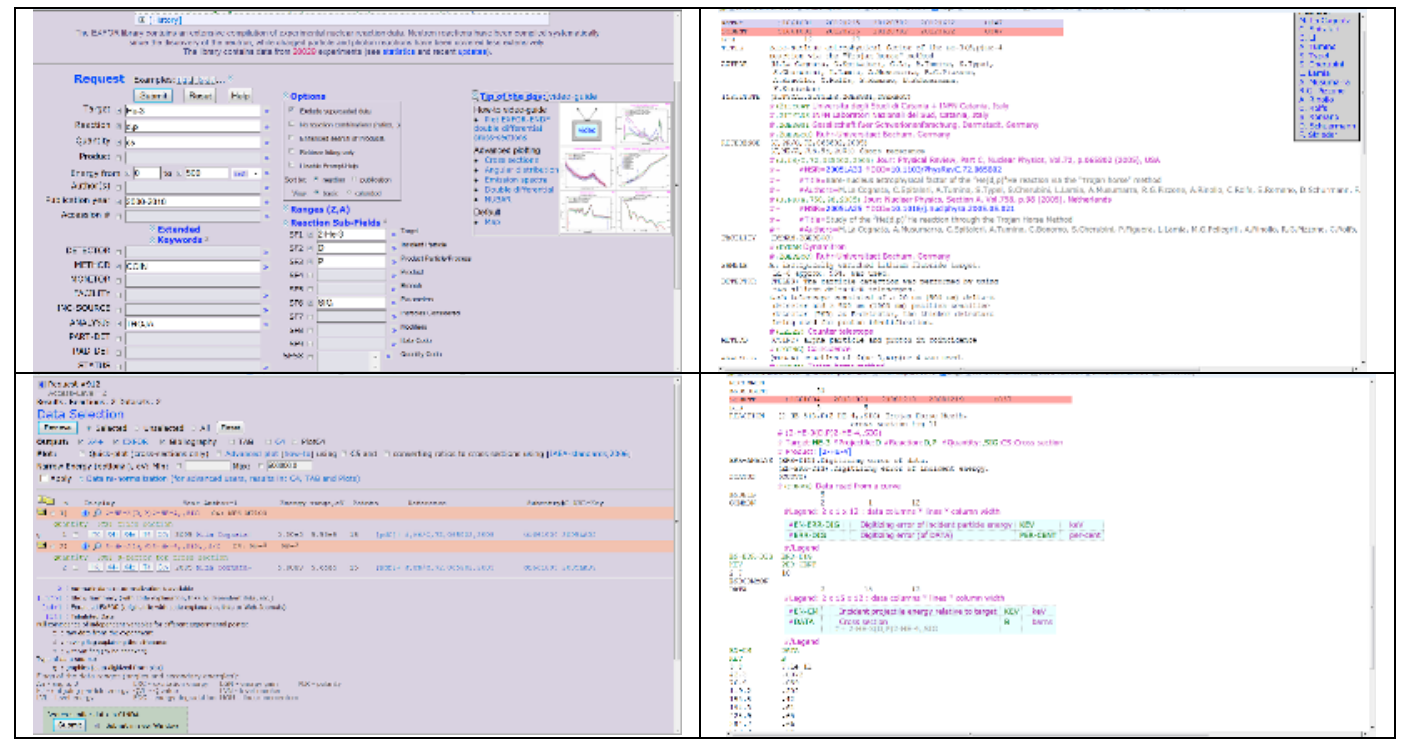

Figure 1. IAEA Nuclear Data Services. EXFOR retrieval webpage (left) and output (right)

The data/information can be retrieved in: extended EXFOR format $(\mathrm{X}+)$, BibTeX, TABLE, BIB and XREF, T4 format, C4 format, extended C4 and R33 format [7]. The data can be displayed on graphs in static and interactive plots with option for uploading of users' data for comparison.

\section{Recent and future developments}

Availability of complete information required for the proper interpretation of the experimental results is essential for the compilation in EXFOR. Many nuclear reaction data, included in the EXFOR database, have been obtained by experiments with neutron sources that have a relatively broad energy (or non-monoenergetic) distribution. Several types of neutron fields applied in spectrum average cross 
section measurements were discussed during the CM "Neutron Sources Spectra for EXFOR" [8] and the neutron spectral distributions provided by authors were compiled with link to the spectrum average cross section data. Neutron-induced reaction cross section data in the resonance range are important for many fields of science and technology. Many facilities employ accelerators for production of neutrons in a broad energy range and data are obtained by transmission and capture measurements by time-of-flight method. Proper analysis of the differential data in the resonance range (total, capture, fission cross sections, and time-of-flight spectra) requires knowledge of response functions of the complete target to detector measurement equipment employed at particular experimental facility. There are on-going activities to collect and include detectors response functions for TOF data in EXFOR.

The gamma scattering $\left(\gamma, \gamma^{\prime}\right)$ with excitation of strong dipole resonances in the nucleus and consecutive prompt $\gamma$-ray decay can be applied for the identification and quantification of the isotopes (stable, radioactive or actinide). A novel non-destructive method for detecting clandestine materials has been developed recently based on Nuclear Resonance Fluorescence (NRF). Compilation of photonuclear scattering reaction data was recently initiated by IAEA NDS.

Beta-delayed neutron emission data are important for nuclear physics, nuclear astrophysics, reactor physics etc. Regarding the characteristics of the delayed neutrons emitted from the fission products total average delayed fission neutron yields are extensively compiled in EXFOR as well as several energy spectra for specific delayed neutron groups. Development of new radioactive-ion beam facilities, mass separators, Penning-trap methods, etc. widen the bounds for measurement of betadelayed neutrons in different mass regions. Inclusion of beta-delay neutron emission data in EXFOR database is considered as beneficial for further experimental investigations, evaluations and theoretical analysis.

\section{Summary}

The member centres of the International Network of Nuclear Reaction Data Centres provide comprehensive collection of experimental nuclear reaction data compilations in its EXFOR data library. The scope and the type of the data evolved and presently neutron-, charged-particle-induced and photonuclear data in the energy range up to $1 \mathrm{GeV}$ are compiled. The database is updated on a monthly basis and at present contains about 20,000 experimental works. Web services for data search and retrieval are provided to users. Different outputs allow retrieval in forms ready for further processing.

\section{References}

1. N. Otuka (ed.), Report IAEA-NDS-207 (Rev.2011/01), IAEA (2011).

2. V. Zerkin, A. Trkov, Proc. Int. Conf. on Nuclear Data for Science and Technology (ND2007), Nice, France, 22-27 Apr., 2007, p. 769 (2008).

3. R.F. Carlson, At. Data Nucl. Data 63, 93, (1996).

4. H. Schopper (ed.), Production of Radionuclides at Intermediate Energies, (Landolt- Börnstein New Series Vol.I/13a-h, Springer (1991-1999)).

5. A.J. Koning (ed.), Report NEA/NSC/WPEC/DOC(2010)/428, OECD NEA (2011).

6. N. Otuka, V. Semkova (eds), Report INDC(NDS)-0629, IAEA (2012).

7. O. Schwerer (ed), Report IAEA-NDS-206, IAEA (2008).

8. S. Simakov, F.Käppeler (eds), Report INDC(NDS)-0590, IAEA (2011). 